\title{
SCIDAR measurements at Pic du Midi
}

\author{
J.-L. Prieur ${ }^{1}$, G. Daigne ${ }^{2}$, and R. Avila ${ }^{3}$ \\ 1 UMR 5572 d'Astrophysique, Observatoire Midi-Pyrénées - Centre National de la Recherche Scientifique, \\ 14 avenue Édouard Belin, 31400 Toulouse, France \\ 2 Observatoire de Bordeaux, BP 89, 33270 Floirac, France \\ e-mail: daigne@observ.u-bordeaux.fr \\ 3 Instituto de Astronomía UNAM, Campus Morelia, A.P. 72-3 (Xangari), 58089 Morelia Michoacán, México \\ e-mail: r.avila@astrosmo.unam.mx
}

Received 2 January 2001 / Accepted 14 February 2001

\begin{abstract}
We present SCIDAR measurements made at the Pic du Midi Observatory with PISCO. A series of vertical profiles of the refractive-index structure constant $C_{\mathrm{N}}^{2}(h)$ was obtained with the observations of the binary stars $\gamma$ Del and $\gamma$ Ari, with 30 second time sampling. In the free atmosphere, the turbulence was dominated by the contribution of a few well-separated atmospheric layers at altitudes in the range 8-20 km, with significant changes within a few minutes. We describe a new method based on the CLEAN algorithm applied on the 2-D covariance function of the irradiance fluctuations in the pupil plane and compare it with the widely-used Maximum Entropy Method applied on a slice of this 2-D function. Our method is much less sensitive to the noise. Vertical profiles of the wind velocity and direction were obtained with an automatic procedure working on the mean cross-correlation function of consecutive frames. Furthermore, we validate a model for the SCIDAR detectivity threshold in terms of distance to the turbulent layers.
\end{abstract}

Key words. atmospheric effects - site testing - turbulence - methods: data analysis - techniques: image processing

\section{Introduction}

A direct estimate of the vertical distribution of turbulence and its time variations can be obtained through the observation of stellar-light irradiance fluctuations in the entrance pupil of a telescope. Indeed, the coherence radius of the scintillation pattern due to a single diffractive layer depends on its distance to the entrance pupil of the telescope, and varies as the square root of this distance. The experiment conducted at Nice Observatory in 1974 by Rocca, Roddier and Vernin (Rocca et al. 1974), on spatio-temporal correlation of scintillations, has shown that the most reliable results can be obtained with the observation of a double star and the so-called spatio-angular correlation of the compound scintillation pattern. Since then, the technique is known as SCIDAR, for SCIntillation Detection And Ranging. It has been used on different telescopes in many astronomical observatories for site testing, as a complement to seeing measurements (for a review, see

Send offprint requests to: J.-L. Prieur,

e-mail: jean-louis.prieur@obs-mip.fr

* Based on observations made with the Télescope Bernard Lyot at Pic du Midi Observatory, France.
Avila 1998). Its spatial resolution along the line of sight cannot compete with in situ measurements for detailed studies of the turbulence distribution, but it may bring some new insights on turbulence lifetime at different altitudes, from a few kilometers upwards.

Our purpose is not site testing, but a preliminary investigation of SCIDAR technique as a support to ground-based differential astrometry. Optical turbulence produced high in the atmosphere will be the main limiting factor in narrow-angle measurements to be performed with dual-field interferometers (DFI) (Shao \& Colavita 1992; Colavita 1994). Its deteriorating effects have to be clearly evaluated before one can try and reduce them. Furthermore, since the SCIDAR technique can be performed with a telescope aperture about the size of the entrance pupil of an optical interferometer observing in the near-IR range ( $D \simeq 1.5 \mathrm{~m}$ ), it could be a unique technique, along with DFI, for probing and tracking turbulence distribution and wind velocity.

Numerous in situ measurements of temperature fluctuations carried out with balloon sounding have shown that regions responsible for optical turbulence are usually localized in a few thin layers, about $10-50 \mathrm{~m}$ thick 
(Coulman et al. 1995). Unfortunately, little (Avila et al. 1998) is known about their temporal variations, on time scales of one minute or so, which are typical integration times for measurements with DFI's in the near IR, see e.g. descriptions of the Palomar Tesbed Interferometer or PTI (Colavita et al. 1999; Lane et al. 2000).

In this paper, we report SCIDAR observations with the Telescope Bernard Lyot (TBL) at Pic du Midi. The $2 \mathrm{~m}$ pupil is large enough for the observation of well separated binary stars, about $10^{\prime \prime}$ apart, so that a spatial resolution of about $1 \mathrm{~km}$ can be achieved at a distance of $10 \mathrm{~km}$. We may recall previous turbulence measurements at Pic du Midi: in the 1980's, it was investigated with the SONATE experiment (Dubosclard et al. 1984; Hecquet \& Klauss 1989), which involved acoustic soundings of the lower atmosphere together with image quality measurements in the visible. Seeing variations were found to be strongly correlated with the turbulence content in the first few hundred meters above the site.

We shall keep in mind that the turbulence content reported here is just a (small) part of the whole optical turbulence above the observatory site. Turbulence in the first few kilometers was not detected, since the observations were not performed in the Generalized SCIDAR mode (Avila et al. 1997; Fuchs et al. 1998).

We first recall the basic principle of SCIDAR measurements in Sect. 2 and describe the observations in Sect. 3. We then describe the analysis of the data and the two methods based on Maximum Entropy Method (MEM hereafter) and CLEAN that we have used to derive $C_{\mathrm{N}}^{2}$ profiles. Results on the atmospheric turbulence, wind velocity profiles and their time variations are presented in Sect. 5 with a detailed analysis of the achieved sensitivity.

\section{The SCIDAR technique}

\subsection{Basic principle with a single turbulent layer}

Classical SCIDAR is based on the observation of irradiance fluctuations of a double star at ground level (Rocca et al. 1974; Caccia et al. 1987; Avila 1998). With a single turbulent layer at distance $d_{j}$ (cf. Fig. 1), two stars with angular separation $\boldsymbol{\theta}^{1}$ cast to the ground similar diffraction patterns shifted by $\boldsymbol{r}_{\boldsymbol{j}}=d_{j} \boldsymbol{\theta}$. The covariance $C_{2}(\boldsymbol{\rho})$ of the irradiance fluctuations exhibit two secondary peaks at $\pm \boldsymbol{r}_{j}$ :

$C_{2}(\boldsymbol{\rho})=A C_{1}(\boldsymbol{\rho})+B\left[C_{1}\left(\boldsymbol{\rho}+d_{j} \boldsymbol{\theta}\right)+C_{1}\left(\boldsymbol{\rho}-d_{j} \boldsymbol{\theta}\right)\right]$

with $A=\left(1+\alpha^{2}\right) /(1+\alpha)^{2}$ and $B=\alpha /(1+\alpha)^{2}$

where $\alpha$ is the brightness ratio of the binary components (related to the magnitude difference $\Delta m$ by $\alpha=$ $\left.10^{-0.4 \Delta m}\right)$ and $C_{1}(\boldsymbol{\rho})$ is the normalized covariance function of the irradiance fluctuations for single star observations. $C_{1}(\boldsymbol{\rho})$ is the Fourier transform of the power

\footnotetext{
${ }^{1}$ In this paper, two-dimensionnal vectors will be noted in bold.
}

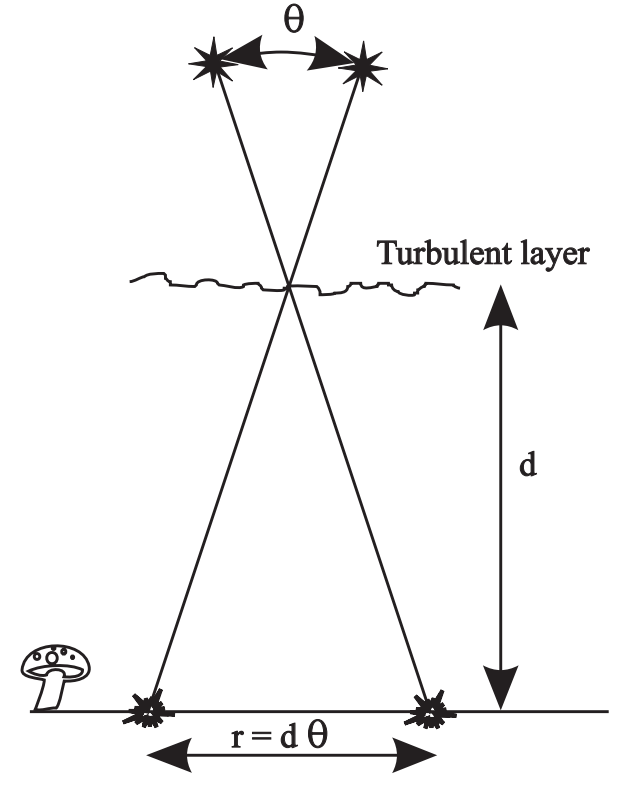

Fig. 1. Schematic of the irradiance fluctuations and their correlation with double star observation, or the SCIDAR basic principle

spectrum $W_{I}$ of the irradiance fluctuations at ground level, given by (Roddier 1981):

$W_{I}(\boldsymbol{f})=4 W_{\Phi}(\boldsymbol{f}) \sin ^{2}\left(\pi \lambda d_{j} f^{2}\right)$

where $\lambda$ is the observed wavelength and $W_{\Phi}$ is the power spectrum for the phase fluctuations at the level of the layer, generally assumed to follow Kolmogorov statistics. In that case $C_{1}(\rho)$ is isotropic and proportional to $J_{j}$, the optical turbulence factor (Tokovinin 1997) of the thin layer, which is related to the refractive index structure parameter, $C_{\mathrm{N}}^{2}(h)$, by:

$J_{j}=\int_{h_{j}-\delta h_{j} / 2}^{h_{j}+\delta h_{j} / 2} C_{\mathrm{N}}^{2}(h) \mathrm{d} h$,

$\delta h_{j}$ being the thickness of the layer. With a line of sight at zenith angle $z$, the observed turbulence factor is $\tilde{J}_{j}=$ $J_{j} \sec (z)$. The expression of $C_{1}(\rho)$ is then:

$C_{1}(\rho)=\tilde{J}_{j} K\left(\rho, d_{j}\right)$

with:

$K\left(\rho, d_{j}\right)=0.243 k^{2} \int_{0}^{\infty} f^{-8 / 3} \sin ^{2}\left(\pi \lambda d_{j} f^{2}\right) J_{0}(2 \pi f \rho) \mathrm{d} f$.

It is plotted in Fig. 2 for different distances to the turbulent layer and was observed in the experiment reported here, with its negative feet. The correlation radius of the irradiance fluctuations is about the radius of the first Fresnel zone $\sqrt{\lambda d / 2 \pi}$ (Tatarskii 1961), so that spatial resolution along the line of sight (i.e., the ability to separate two turbulent layers of equal $J$ factor) is given by:

$\Delta d_{j} \simeq \frac{0.78 \sqrt{\lambda d_{j}}}{\theta}$.

We derived the value of 0.78 from the $F W H M$ (Full Width at Half Maximum) of the curves $C_{1}(\rho)$ in Fig. 2. 


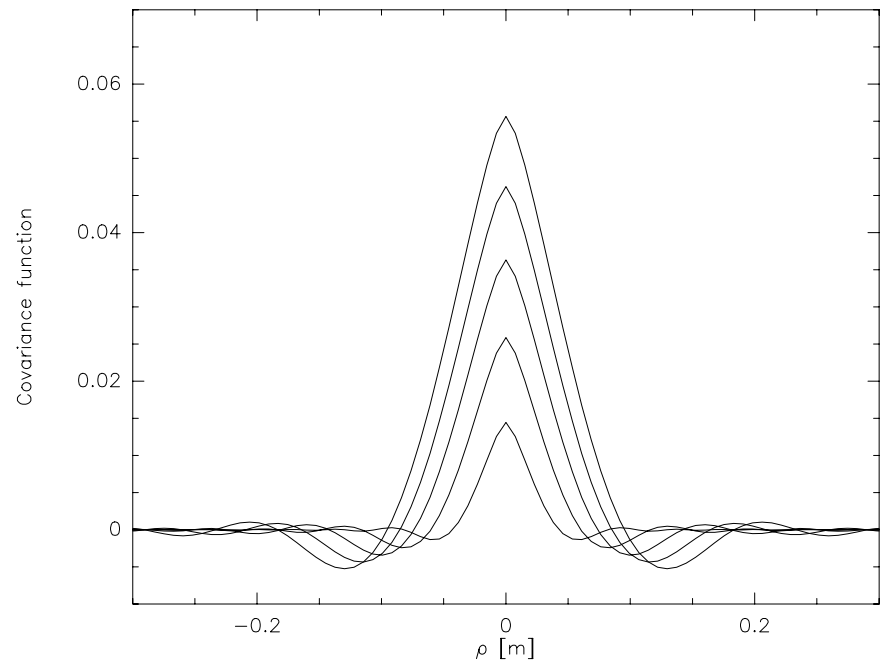

Fig. 2. Covariance function $C_{1}(\rho)$ of irradiance due to a single turbulent layer, with optical turbulence factor $J=$ $3.10^{-14}\left[\mathrm{~m}^{1 / 3}\right]$, at different distances: $h=5,10,15,20,25 \mathrm{~km}$. The wavelength is $\lambda=0.53 \mu \mathrm{m}$

\subsection{Principle with a vertical distribution of turbulence}

Several turbulent layers along the line of sight are statistically independent, so that the different contributions will sum in quadratic form. More precisely, the power spectra of the relative irradiance fluctuations $\delta I / I$ will add (Tatarskii 1961; Roddier 1981). In SCIDAR measurements, only a few turbulent layers will contribute to the covariance function at a given $\rho$ value, away from the central peak, so that the weak-scintillation approximation can be safely used: the covariance function $C_{\text {sci }}(\boldsymbol{\rho})$ outside the central peak is estimated from irradiance fluctuations instead of from the logarithm of the irradiance. With a continuous distribution of turbulence along the line of sight, the covariance profile in the $\theta$ direction will be:

$C_{\mathrm{sci}}(\rho)=B \sec (z) \int_{0}^{\infty} K(\rho, h \sec (z)) C_{\mathrm{N}}^{2}(h) \mathrm{d} h$

which is a Fredholm-type integral with kernel $K(\rho, h \sec (z))$. A maximum entropy method (MEM) can be used to recover the distribution of the index structure parameter (Avila 1998).

An alternative is to consider a multi-layer model with a discrete distribution of turbulence along the line of sight:

$C_{\text {sci }}(\boldsymbol{\rho})=B \sum_{j} \tilde{J}_{j} K\left(\left|\boldsymbol{\rho}-\boldsymbol{r}_{j}\right|, d_{j}\right)$

which can be solved in 2-D space with a CLEAN algorithm. In Sect. 5, we shall compare the results obtained with one and the other method.

\subsection{Limited pupil size and sensitivity}

In practice, the covariance function is estimated with a limited pupil size, and the incoming radiation has to be multiplied by a pupil function $P(\boldsymbol{r})(P=0$ or 1$)$.
Experimentally, the normalized covariance function of irradiance $C_{2}(\boldsymbol{\rho})$ will be approximated with:

$C_{2}(\boldsymbol{\rho}) \approx C_{\delta I}(\boldsymbol{\rho}) / C_{I_{0}}(\boldsymbol{\rho})$

where $C_{I_{0}}(\boldsymbol{\rho})$ is the covariance of the average pupil irradiance function $I_{0}(\boldsymbol{\rho})$ estimated on a large ensemble of frames and $C_{\delta I}(\boldsymbol{\rho})=\left\langle\left[I(\boldsymbol{\rho})-I_{0}(\boldsymbol{\rho})\right]^{2}\right\rangle$ is the covariance of the irradiance fluctuations, per frame, relative to $I_{0}(\boldsymbol{\rho})$. Let us now estimate the expected limiting sensitivity of our measurements. The statistical rms noise of $C_{\text {sci }}(\rho)$, per frame is given by (Tokovinin 1997):

$\Delta C_{\mathrm{sci}}(\rho)=\left(C_{2}(0)+1 / N_{\mathrm{ph}}\right) / \sqrt{M(\rho)}$

where $N_{\text {ph }}$ is the mean number of photons per coherence area and $C_{2}(0)$ is the intensity dispersion $\left(=A \sigma_{I}^{2}\right.$, where $\sigma_{I}^{2}$ is the scintillation index). The read-out noise and/or recording noise are not considered here. The number of independent cells $M(\rho)$ is given by the ratio of the overlapping pupil area $S$ to the coherence area of the scintillation patterns. Following the detailed analysis performed by Vernin \& Azouit (1983), we take it as the Fresnel zone area $\left(\lambda d_{0} / 2\right)$ where $d_{0}$ is the distance of the dominant turbulent layer which governs the size of the noisy structures in the covariance function. With circular aperture of diameter $D$, the overlapping pupil area is $S(x)=f(x) D^{2} / 2$ with $x=\rho / D$ and $f(x)=\arccos (x)-x\left(1-x^{2}\right)^{1 / 2}$. Hence:

$M(\rho)=\frac{D^{2} f(x)}{\lambda d_{0}}$.

From Eq. (9) applied at $\rho=r_{j}$, the location of the maximum of the $j$ th component, we deduce the expression of the uncertainty of the optical turbulence factor, for the layer at distance $d_{j}$ :

$\Delta \tilde{J}_{j}=\frac{\Delta C_{\mathrm{sci}}\left(r_{j}\right)}{B K\left(0, d_{j}\right)}$

With $K\left(0, d_{j}\right)=19.12 \lambda^{-7 / 6} d_{j}^{5 / 6}$, we obtain a general expression for the rms noise per frame in SCIDAR measurements:

$\Delta \tilde{J}_{j}=\frac{5.2310^{-2} \lambda^{5 / 3} d_{j}^{-5 / 6} d_{0}^{1 / 2}\left(C_{2}(0)+1 / N_{\mathrm{ph}}\right)}{B D \sqrt{f(x)}}$

with $x=\theta d_{j} / D$. For $N$ independent frames, $\Delta \tilde{J}_{j}$ is multiplied by $N^{-1 / 2}$. It is plotted in Fig. 3 in terms of the distance $d_{j}$ to the turbulent layer for $N=1500, B=0.25$ (i.e., components of equal magnitude) and $\lambda=0.53 \mu \mathrm{m}$. The coefficients $\left(C_{2}(0)+1 / N_{\mathrm{ph}}\right)=0.15$ and $d_{0}=17 \mathrm{~km}$ are close to the measured values with our observations (Sect. 5.3). Hence with aperture diameters larger than $1.5 \mathrm{~m}$, the altitude range above $\sim 9 \mathrm{~km}$ can be probed with an uncertainty smaller than $10^{-15}\left[\mathrm{~m}^{1 / 3}\right]$ for the turbulence factor. 


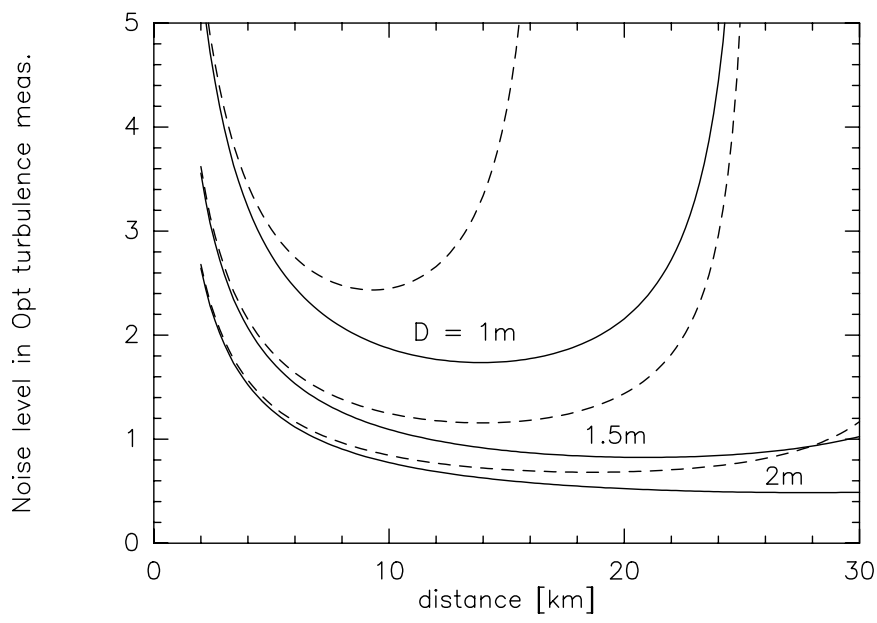

Fig. 3. Noise level (rms) in SCIDAR measurements of the optical turbulence factor $J$ (in $10^{-15} \mathrm{~m}^{1 / 3}$ ), with different circular apertures, whose diameter $D$ is in the range 1-2 $\mathrm{m}$. The angular separation of the observed binary star is $8^{\prime \prime}$ (solid line), or $12^{\prime \prime}$ (dashed line)

\section{Observations}

\subsection{Instrumental setup}

The observations were carried out with the PISCO $^{2}$ speckle camera (Prieur et al. 1998), developed by Observatoire Midi-Pyrénées and operated at the Cassegrain Focus of the 2-meter Télescope Bernard Lyot (TBL) of the Pic du Midi Observatory (altitude: $2880 \mathrm{~m}$ ). It is a remotely controlled versatile instrument that can be configured in various observing modes during the observations (i.e., direct imaging, spectroscopy and pupil imaging) which provides a powerful tool for investigating the field of close binary stars, as already shown in previous publications (Aristidi et al. 1999; Scardia et al. 2000; Prieur et al. 2000).

The PISCO speckle camera was used in its pupil imaging mode with the ICCD (intensified CCD with a multichannel plate) of Nice University (Aristidi et al. 1997). An example of the pupil image is given in Fig. 4. The oval shape is due to an internal diaphragm that was left there by error. The scale on the output digitized $284 \times 388$ pixel images was $0.763 \mathrm{~cm} /$ pixel in the pupil plane which corresponds to a scale of distance $d=r / \theta$ of $208.5 \mathrm{~m} /$ pixel for $\gamma$ Ari and $141.8 \mathrm{~m} /$ pixel for $\gamma$ Del along the line of sight.

For the orientation of the detector, we used the calibration done for a program of speckle observations during the same night (Scardia et al. 2000).

\subsection{Observed stars}

The binaries best suited for our purpose in September were $\gamma$ Del and $\gamma$ Ari (cf. Table 1), with a small brightness difference and bright enough to be detected by the ICCD in the pupil image with a short exposure time. We also

\footnotetext{
${ }^{2}$ PISCO stands for "Pupil Interferometry Speckle camera and COronagraph".
}

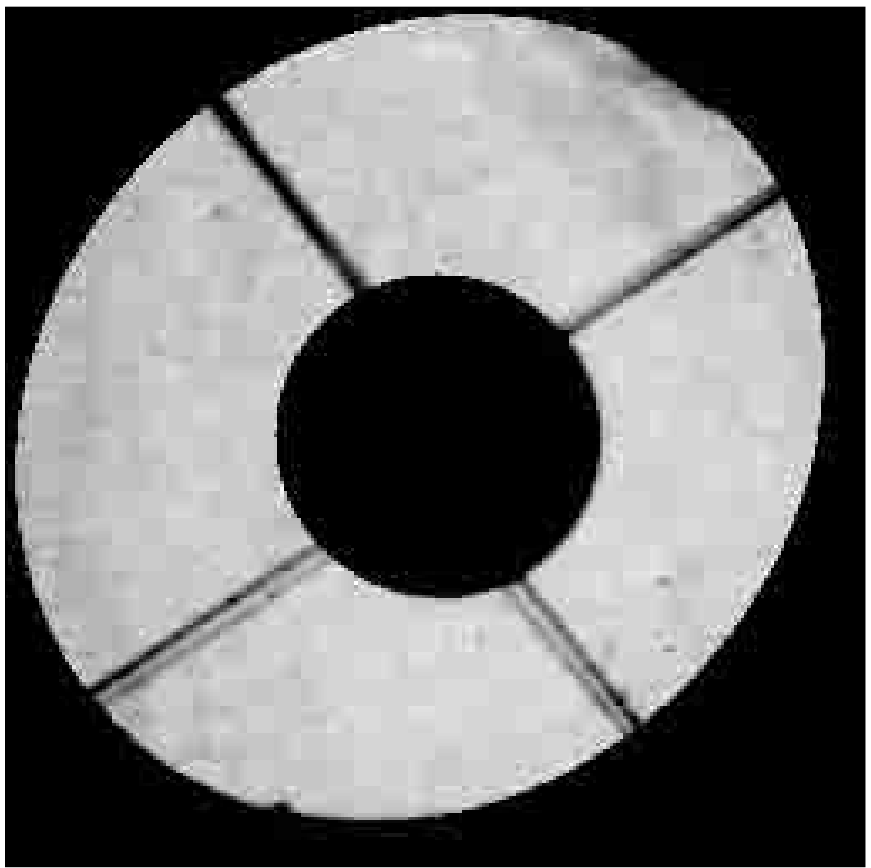

Fig. 4. Image of the pupil

observed single stars ( $\alpha$ Del and $\left.\beta \mathrm{Ari}^{3}\right)$ as a reference for testing possible sources of noise and systematic errors.

\subsection{Observing conditions}

Binary star observations were performed during two short periods during the night of 5-6th Sep. 1998, with a seeing value of $\sim 1.5^{\prime \prime}$ and some atmospheric absorption, especially during $\gamma$ Del observations. Data from the weather station (Table 2) show an unusual temperature rise at ground level during that night because of the arrival of a perturbation from the West (see Sect. 5.2).

\section{Data processing and analysis}

\subsection{Pre-processing}

The SVHS tapes recorded during the observations were digitized by a specially-designed $\mathrm{C}++$ program which controls a Video Cassette Recorder (VCR hereafter) Panasonic AG-7355 with a RS232 link and a digitizing board (Ellips/Full Rio) connected to the PCI bus of a Penthium MMX $166 \mathrm{MHz}$. Sets of 200 frames were processed at a rate of 50 frames/s and buffered to the RAM memory of the PC to allow for the computation of the mean normalized covariance function as defined in Eq. (10). This step was rather lengthy (a few hours) due to the number of elementary frames ( 15000 for $\gamma$ Del).

\footnotetext{
3 Although $\beta$ Ari is a spectroscopic binary, the two components are close enough to be considered as a single star in scintillation measurements.
} 
Table 1. List of observed objects. $V$ : total magnitude, Elev.: elevation above the horizon, $\tau$ : integration time of elementary frames

\begin{tabular}{|cccccccl|}
\hline Name & HD number & $V$ & Date & $\begin{array}{c}\text { Time } \\
(\mathrm{U} . T .)\end{array}$ & $\begin{array}{c}\text { Elev. } \\
{ }^{\circ}\end{array}$ & $\begin{array}{c}\tau \\
(\mathrm{msec})\end{array}$ & Comments \\
\hline$\gamma$ Del & HD 197963-64 & 3.9 & $05 / 09 / 98$ & $22 \mathrm{~h} 46$ & 60.4 & 2 & $\theta=11^{\prime \prime} 1$, posi. angle: $268^{\circ}, \Delta_{m}=0.7$ \\
$\alpha$ Del & HD 196867 & 3.8 & $05 / 09 / 98$ & $21 \mathrm{~h} 52$ & 62.9 & 2 & Reference for $\gamma$ Del \\
$\gamma$ Ari & HD 11502-03 & 3.9 & $06 / 09 / 98$ & $3 \mathrm{~h} 00$ & 67.8 & 3 & $\theta=7^{\prime \prime} 55$, posi. angle: $0.7^{\circ}, \Delta_{m}=0.05$ \\
$\beta$ Ari & HD 11636 & 2.6 & $06 / 09 / 98$ & $3 \mathrm{~h} 02$ & 67.8 & 3 & Reference for $\gamma$ Ari \\
\hline
\end{tabular}
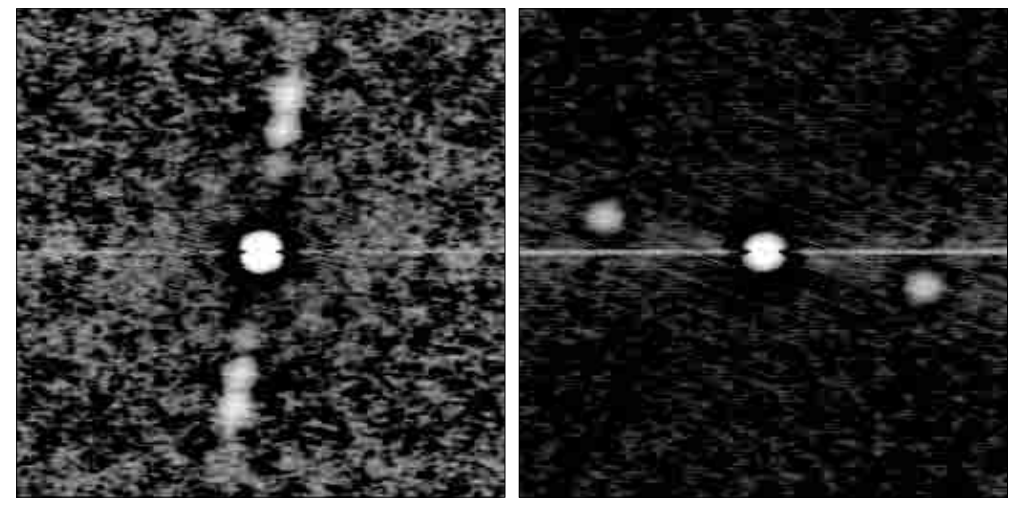

Fig. 5. Examples of $C_{2}(\boldsymbol{\rho})$ for $\gamma$ Ari (left) and $\gamma$ Del (right). North is in the upper part of the image, East to the left. The central bright line is an artefact due to the video scanning

\subsection{Experimental $C_{2}(\rho)$ functions}

For $\gamma$ Ari and $\gamma$ Del, the $C_{2}(\boldsymbol{\rho})$ functions exhibit an elongated knotty pattern aligned along the direction of the line joining the two components of the double star (cf. Fig. 5). The central peak and the central line are perturbed by a read-out noise which shows a correlation between pixels along the lines. Fortunately, this did not affect the SCIDAR measurements since they were done outside this peak (cf. Sect. 2.2). Another instrumental contribution was an oblique fringed pattern in $C_{2}(\boldsymbol{\rho})$ which was removed by Fourier filtering. This video noise appears when the level of the intensity is very low, as it is the case here because of the very short exposure time $(\tau<3 \mathrm{msec})$. This noise was larger in the case of $\gamma$ Del, for which the intensity level was lower and $\tau=2 \mathrm{msec}$. It is probably due to interferences of the line frequency of the camera and/or the VCR when recording the data.

To verify the robustness of our results we performed the following tests:

(i) we changed the parameters of the digitization of the frames. It appeared that the results were stable and that the standard set of parameters was optimal in terms of noise (Contrast $=70$, Brightness $=140$ for the Ellips/RIO card);

(ii) we only used a subset of the data by putting a smaller square mask on the pupil: the results were more noisy but very similar;

(iii) we also performed the same processing on the data of the single star $\beta$ Ari, which did not exhibit any significant pattern.
Table 2. Measurements made at the weather station of Pic du Midi at the ground level. The temperature (Col. 2) is in degrees Celsius. The direction orientation (Col. 3) is such that North $=360^{\circ}$ and West $=270^{\circ}$

\begin{tabular}{|ccccc|}
\hline $\begin{array}{c}\text { Time } \\
\text { (U.T.) }\end{array}$ & $\begin{array}{c}T \\
{ }^{\circ} \mathrm{C}\end{array}$ & $\begin{array}{c}\text { Wind direction } \\
\circ\end{array}$ & \multicolumn{2}{c|}{$\begin{array}{c}\text { Wind speed } \\
(\mathrm{m} / \mathrm{s})\end{array}$} \\
& & & $(\mathrm{mean})$ & $(\mathrm{maxi})$ \\
\hline $21 \mathrm{~h} 00$ & 4.9 & 330 & 12 & 14 \\
$22 \mathrm{~h} 30$ & 5.2 & 310 & 6 & 12 \\
$23 \mathrm{~h} 00$ & 6.5 & 330 & 9 & 14 \\
$00 \mathrm{~h} 00$ & 7.4 & 310 & 7 & 13 \\
$03 \mathrm{~h} 00$ & 8.6 & 270 & 9 & 14 \\
$06 \mathrm{~h} 00$ & 9.2 & 280 & 9 & 8 \\
$08 \mathrm{~h} 00$ & 11.0 & 270 & 11 & 14 \\
\hline
\end{tabular}

\subsection{Derivation of the $C_{N}^{2}$ profile with MEM}

Theoretically, all the information needed to retreive $C_{\mathrm{N}}^{2}(h)$ is contained in a radial section of $C_{2}(\rho)$, along the double star separation (cf. Sect. 2.2). In order to eliminate the central peak which contains the uncorrelated noise and where the contribution of each layer is undistinguishable from that of the others, since they are added, we compute the difference of the sections of the measured autocorrelation function parallel and perpendicular to the star separation, $C_{2}^{\|}(\rho)$ and $C_{2}^{\perp}(\rho)$ respectively. This quantity can be written as Eq. (8) plus an additive noise function $N(\rho)$ :

$C_{2}^{\|}(\rho)-C_{2}^{\perp}(\rho)=C_{\text {sci }}(\rho)+N(\rho)$. 

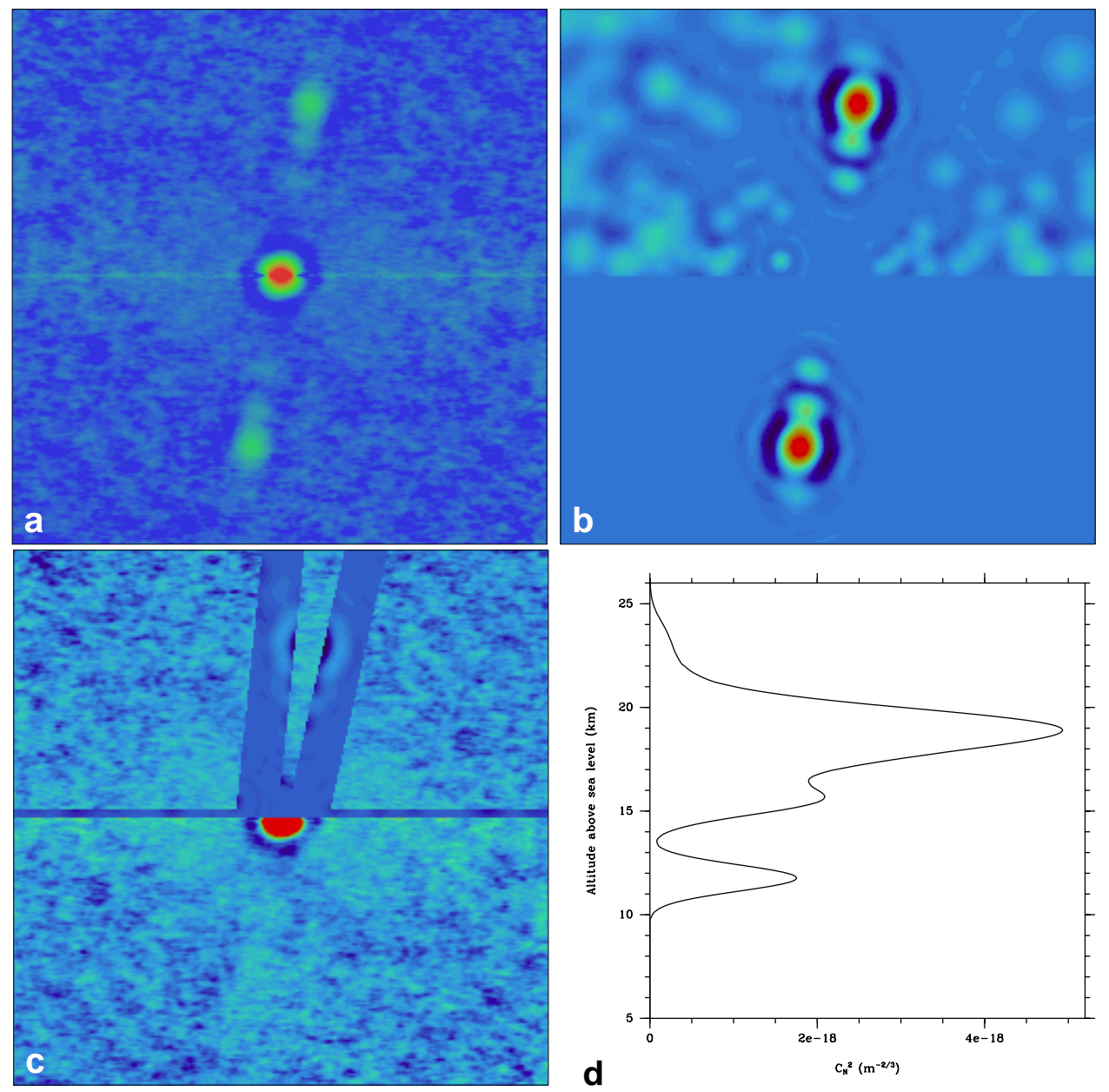

Fig. 6. Example of $C_{\mathrm{N}}^{2}$ inversion of $C_{2}(\boldsymbol{\rho})$ function a) of $\gamma$ Ari with the CLEAN derived method. b) clean image; c) residuals; d) restored $C_{\mathrm{N}}^{2}$ profile. For b) and $\mathbf{c}$ ), the upper part shows all the clean components within the "binary" and the "test" zone, the bottom part shows the components selected within the "binary" zone only

The resulting equation is of Fredholm type and can be inverted by finding a solution that maximizes the joint entropy of the unknown quantities $C_{\mathrm{N}}^{2}(h)$ and $N(\rho)$. The entropy is calculated analytically. Its maximization, using the Lagrange multipliers method, leads to a system of non-linear equations, which is solved by a Newtonian iterative algorithm. In each iteration, an auto-correlation function is calculated from the retrieved $C_{\mathrm{N}}^{2}(h)$ and $N(\rho)$. The iterations are stopped when the difference between this calculated auto-correlation and the measured one has a variance which is less than the estimated variance of the noise. This method was originally developed by (Vernin 1984). The program used here was written by J. Vernin and R. Avila (Avila 1998).

\subsection{Derivation of the $C_{N}^{2}$ profile with CLEAN}

We have written a new program in $\mathrm{C}$ based on the CLEAN algorithm which takes advantage of the 2-D information in order to improve the robustness of the inversion.

For each step $k$, it looks for the location $\boldsymbol{\rho}_{k}$ and value $z_{k}$ of the maximum in the $2-\mathrm{D}$ residual map, then computes $d_{k}$, its corresponding distance along the line of sight, and subtracts a fraction of the 2-D function $C_{\text {sci }}\left(\boldsymbol{\rho}, d_{k}\right)=B \tilde{J}_{k} K\left(\boldsymbol{\rho}, d_{k}\right)$ from the residual map, with:

$$
\tilde{J}_{k}=\frac{z_{k}}{19.12 \lambda^{-7 / 6} d_{k}^{5 / 6} B}
$$

where $B=0.25$ for $\gamma$ Ari, and $B=0.23$ for $\gamma$ Del (cf. Eq. (1)). The maxima are searched within two regions of the $C_{2}(\boldsymbol{\rho})$ image: the "binary zone", i.e., a sector centered on the direction joining the two components of the binary and whose opening angle is ajusted to the width of the covariance function $C_{\text {sci }}(\rho)$ (cf. Fig. 2), and the "test zone", i.e. a region further out (see Fig. 6c) which is used to determine the noise level of the image. When this noise level is reached, the process is stopped. It then outputs a CLEAN map (Fig. 6b), a final residual map (Fig. 6c) and a list of components $\left(\tilde{J}_{k}\right)$.

We then follow the general scheme used in radioastronomy which convolves the CLEAN components by the "CLEAN beam", generally Gaussian, to generate the CLEAN map (Schwarz 1978). The final $C_{\mathrm{N}}^{2}$ profile is obtained by the convolution of the list $\left(\tilde{J}_{k}\right)$ with a set of normalized Gaussian functions centered at $d_{k}$ whose sigma is 

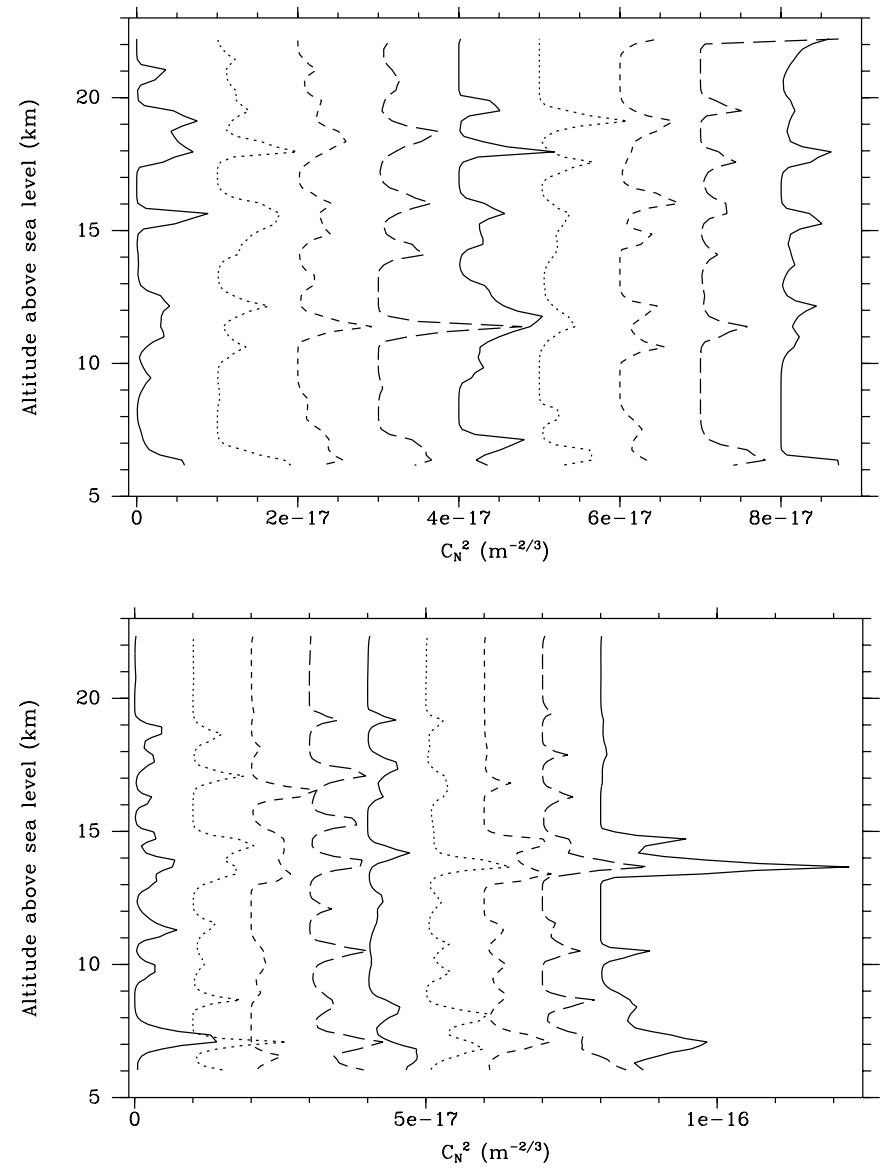

Fig. 7. Set of $C_{\mathrm{N}}^{2}$ profiles computed with $\gamma$ Ari (top) and $\gamma$ Del (bottom) (every 30 seconds) with MEM

equal to the resolution $\Delta d_{k}$ of the SCIDAR measurements at this distance $d_{k}$ :

$C_{\mathrm{N}}^{2}(d) \approx \sum_{k} \tilde{J}_{k} \times \frac{1}{\Delta d_{k} \sqrt{2 \pi}} \exp \left(-\frac{\left(d-d_{k}\right)^{2}}{2 \Delta d_{k}}\right)$

with $\Delta d_{k}=0.34 \sqrt{\lambda d_{k}} / \theta$ (cf. Eq. (7) which gives $\Delta_{j}$ the $F W H M$, i.e., $2.3 \sigma$ of a Gaussian). One could object that this operation degrades the resolution, but (i) this convolution is part of the CLEAN algorithm and regularizes it and (ii) it takes into account the physics governing the SCIDAR detection, more precisely, the Fresnel propagation (cf. Sect. 2). A higher resolution as in the case of our current implementation of MEM (cf. Sect. 5) would actually be a "super-resolution" relative to the data and possibly subject to artefacts.

\section{Results and discussion}

\section{1. $C_{N}^{2}(h)$ profiles}

A set of $9 C_{\mathrm{N}}^{2}$ profiles was computed for each binary star observation with MEM (Fig. 7) and CLEAN (Fig. 8). Each profile corresponds to a sequence of 30 seconds, and the sequences are contiguous. We have checked that both programs were consistent in the case of good signal to noise ratio (SNR) and in particular lead to similar values of the
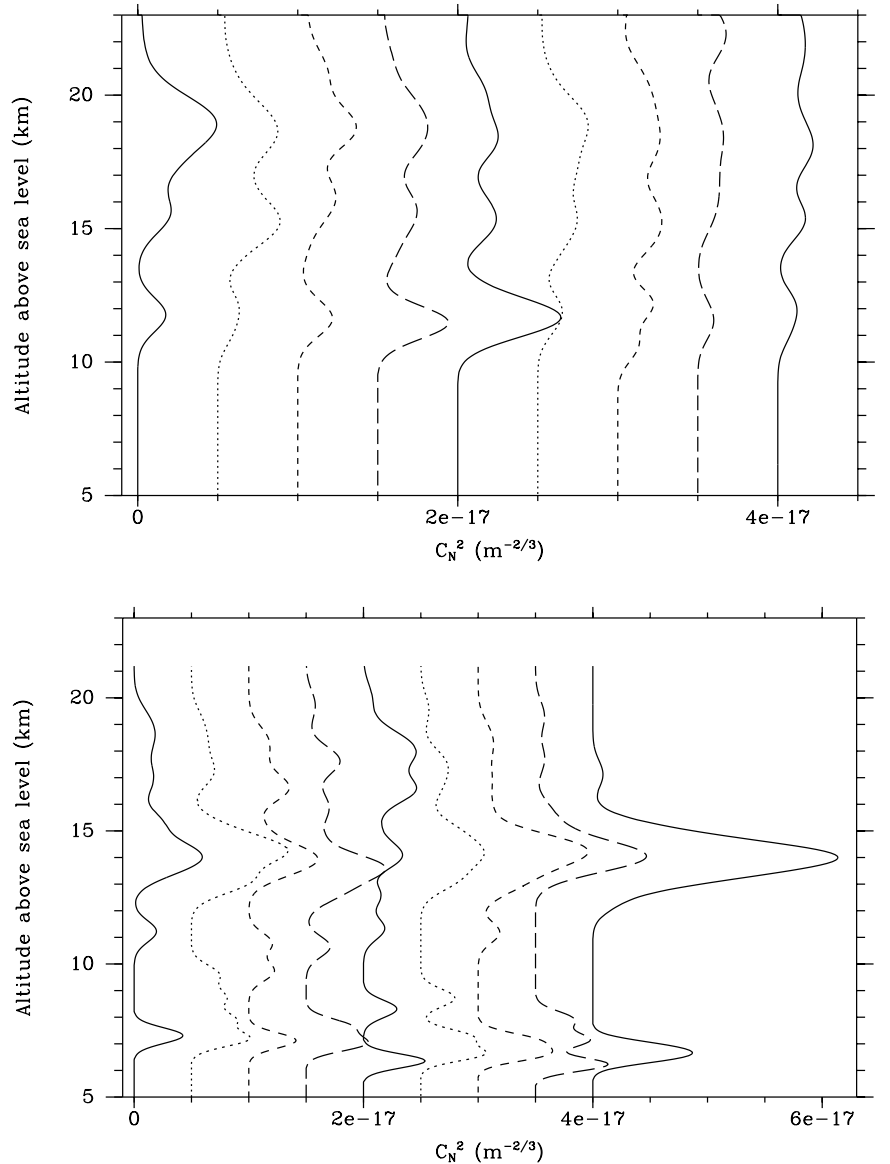

Fig. 8. Set of $C_{\mathrm{N}}^{2}$ profiles computed with $\gamma$ Ari (top) and $\gamma$ Del (bottom) (every 30 seconds) with CLEAN

integral $\int C_{\mathrm{N}}^{2}(h) \mathrm{d} h$ : we found an agreement within $\sim 4 \%$. In the case of faint $C_{\mathrm{N}}^{2}$ components, these programs lead to slightly different results (Figs. 7 and 8).

The MEM program provides a higher resolution in altitude and (consequently) more noisy results. This method is known to lead to slightly "over-resolved" structures. Another reason for a higher noise level is that our current implementation of MEM works on a slice of the $C_{2}(\boldsymbol{\rho})$ function, whereas our CLEAN program operates on the full 2-D $C_{2}(\boldsymbol{\rho})$. A future improvement of the MEM program will consist of extending it to a full 2-D analysis.

In Figs. 7 and 8 we see a rapid change in the turbulence content in the case of $\gamma$ Ari observations. During the first 3 min the uppermost turbulent layer at $h=19 \mathrm{~km}$ fades, whereas the layer at $12 \mathrm{~km}$ becomes more turbulent and dominates the whole profile. It then decreases leading to a quiet featureless turbulent profile. If we take into account the big difference of the wind velocity of these two layers $(\Delta v \sim 40 \mathrm{~m} / \mathrm{s}$ as we shall see in Sect. 5.2), we understand that a significant change would have had occurred for observations with an adaptive optics system, for instance, in terms of temporal anisoplanetism (Klückers et al. 1998). This example shows the interest of a continuous monitoring of the turbulence profile to improve the performance of adaptive optics for very large telescopes or interferometers. 


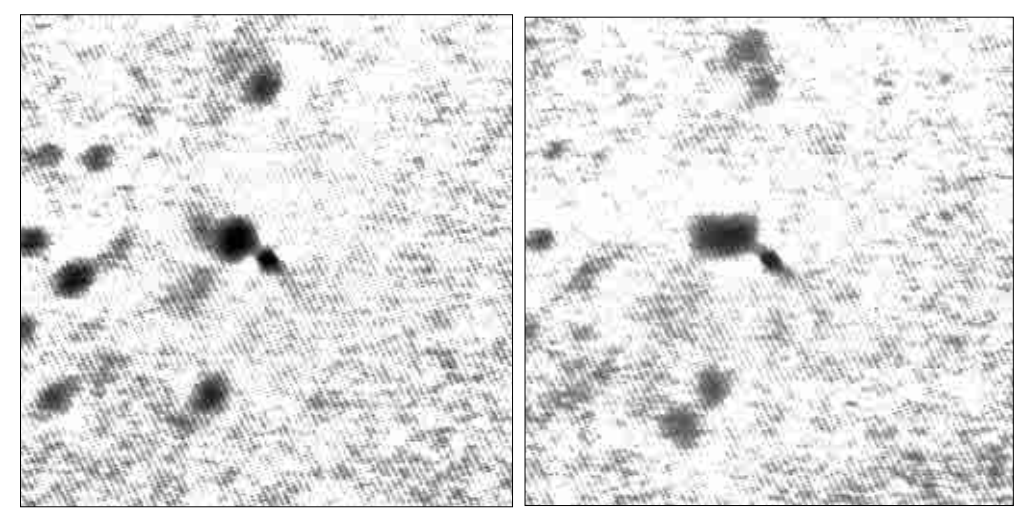

Fig. 9. $C_{2}^{\mathrm{w}}(\boldsymbol{\rho})$ of $\gamma$ Ari with $\Delta t=20$ msec: wind determination at two moments separated by $2 \mathrm{~min}$
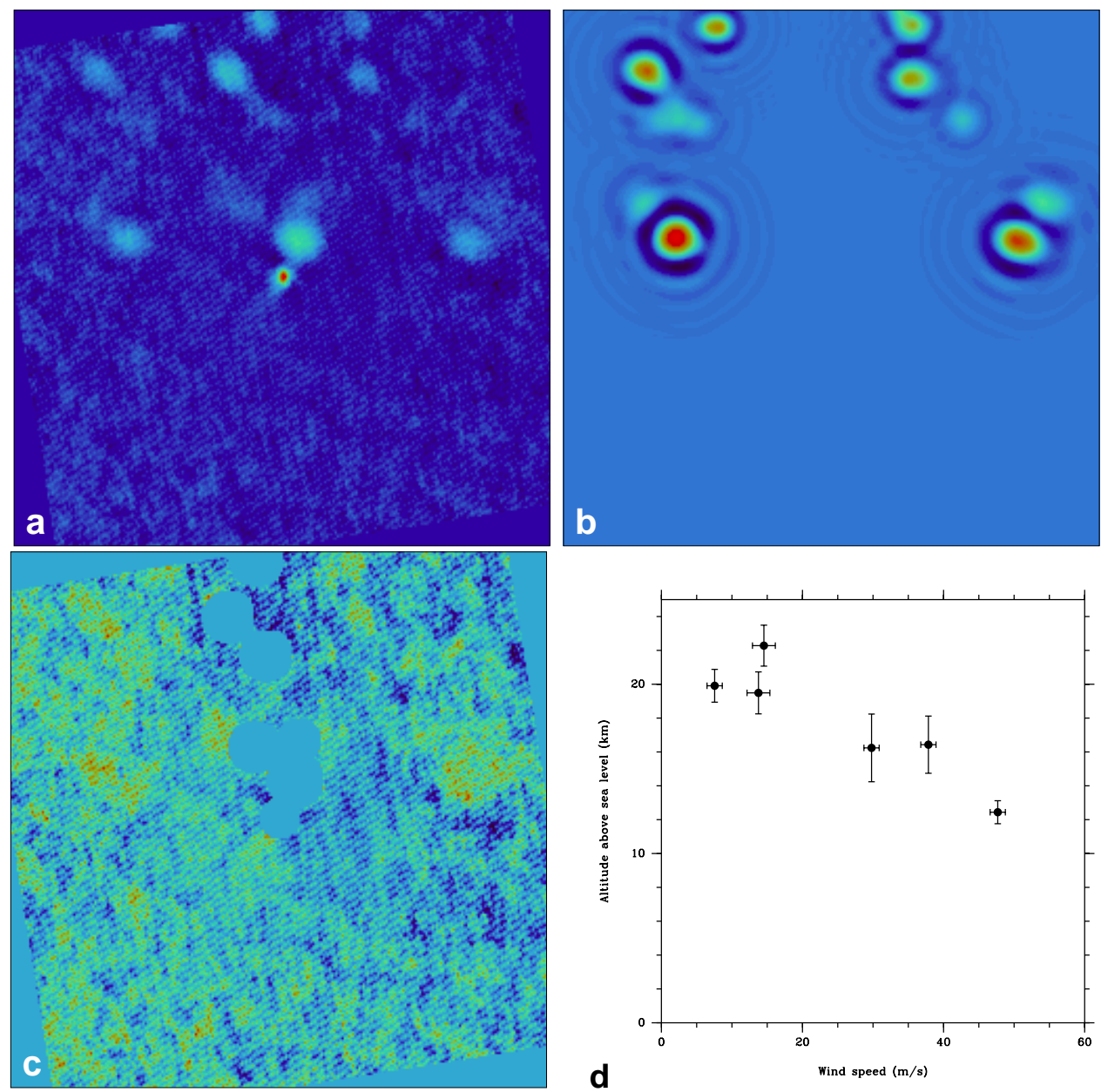

Fig. 10. Example of wind measurement with a CLEAN derived method: a) initial cross-correlation image $C_{2}^{\mathrm{w}}(\boldsymbol{\rho})$ (rotated to align the two components of the binary with the $O x$ axis); b) clean image; c) residuals; d) restored wind velocity profile

The profiles obtained with $\gamma$ Del do not show such dramatic changes and are dominated by a turbulent layer at $h=14 \mathrm{~km}$. The lower part of the curves (below $\sim 8 \mathrm{~km}$ ) should be taken with cautious since the conventional SCIDAR technique is limited to rather high altitudes.

\subsection{Profiles of wind velocity and direction}

The wind velocity and direction were derived from the analysis of $C_{2}^{\mathrm{w}}(\boldsymbol{\rho})$ :

$$
C_{2}^{\mathrm{w}}(\boldsymbol{\rho})=\frac{C(\boldsymbol{\rho}, \Delta t)-C_{I_{0}}(\boldsymbol{\rho})}{C_{I_{0}}(\boldsymbol{\rho})}
$$



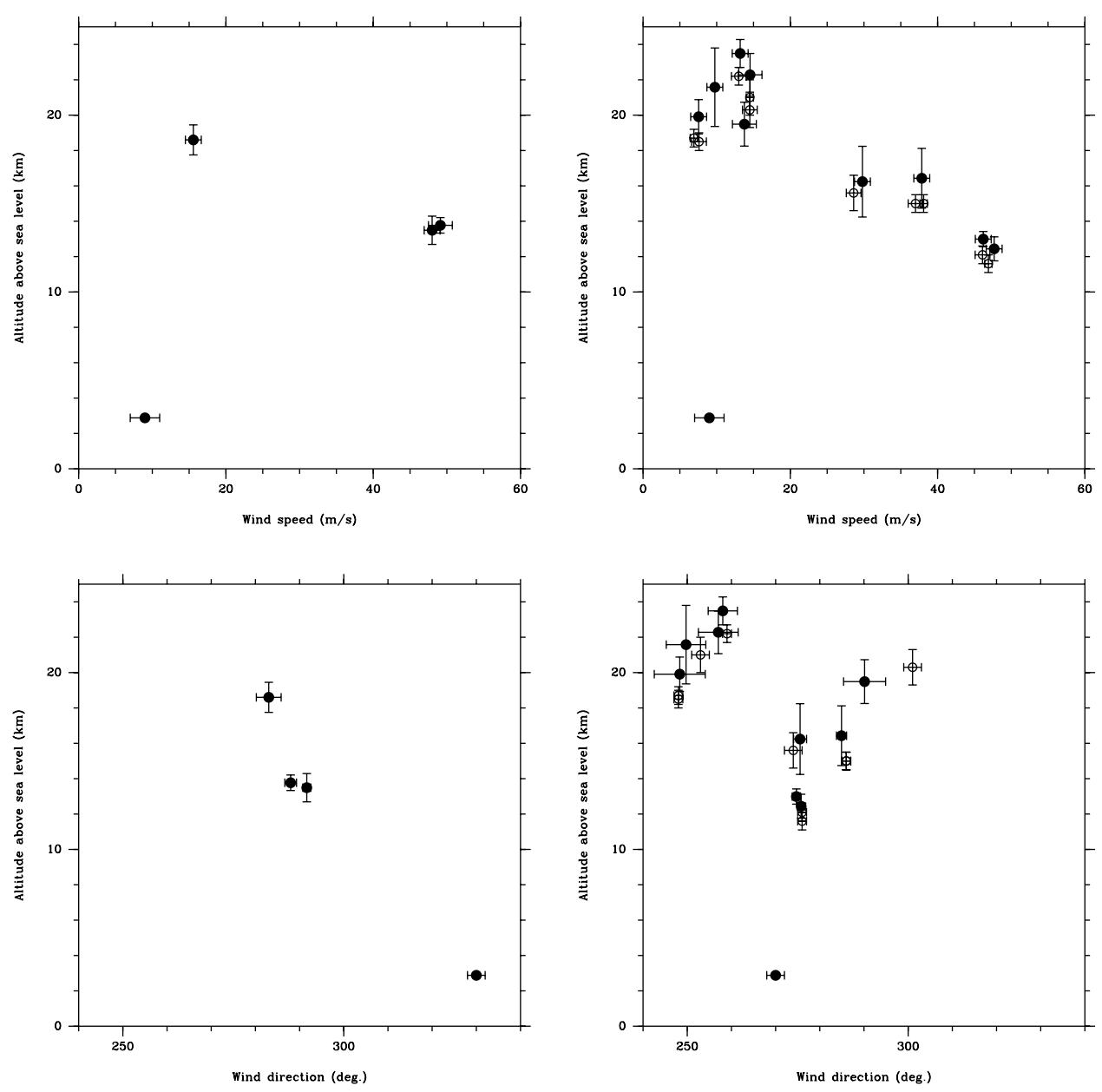

Fig. 11. Vertical wind velocity (top) and direction (bottom) profiles measured with $\gamma$ Del at 23 h00 U.T. (left) and $\gamma$ Ari at 3h00 U.T. (right). Filled circles: automatic determination, open circles: interactive. The lowest point was provided by ground measurements (cf. Table 2). Note the significant change of the wind direction at the ground level due to the arrival of a perturbation

where $C(\boldsymbol{\rho}, \Delta t)$ is the mean cross-correlation of the irradiance function of two successive frames (separated with $\Delta t=20 \mathrm{msec}$ ) and $C_{I_{0}}(\boldsymbol{\rho})$ the covariance of the mean images (cf. Eq. (10)). Examples of experimental $C_{2}^{\mathrm{w}}(\boldsymbol{\rho})$ functions are shown in Fig. 9. Each turbulent layer produces triplets, as in the case of $C_{2}(\boldsymbol{\rho})$, but these triplets are translated from the center because of the wind (Taylor's hypothesis of "frozen" turbulence transported by the wind). This shift allows the measurement of the wind velocity and direction, whereas the distance of the furthest components gives the altitude of the layer. This method was proposed by Avila (1998) and Klückers et al. (1998) and was recently shown to give very reliable velocity measurements on the whole upper atmosphere, in full agreement with simultaneous balloon measurements (Avila et al. 2001).

We have developed a program based on the CLEAN algorithm to measure the wind velocity and direction on these cross-correlation functions. This program looks for "triplets" aligned with the direction of separation of the binary. For each triplet, the center of the central patch is accurately determined by fitting a Gaussian to this patch and the two other components are "cleaned" by removing covariance functions $C_{1}(\rho)$ (Fig. 2), following the same iterative process as described in Sect. 4.4. An example of such a reduction process is given in Fig. 10, whereas the whole profiles obtained from our measurements are given in Fig. 11. For $\gamma$ Ari the mean direction of the wind was nearly perpendicular to the separation of the two stars, which facilited the measurements, whereas these two directions were nearly parallel for $\gamma$ Del. This (and a worse SNR of the data) explains the smaller number of measurements in that case.

The velocity profiles are similar for $\gamma$ Ari and $\gamma$ Del and in good agreement with the profiles obtained at Cerro Tololo by Avila et al. (2001). In our case we note the presence of high velocity winds at an altitude of $\sim 13 \mathrm{~km}$ with speeds of $\sim 50 \mathrm{~m} / \mathrm{s}$, which is larger than the maximum of $\sim 40 \mathrm{~m} / \mathrm{s}$ observed by Avila et al. (2001).

As shown by the ground level measurements (Table 2), a hot front was coming from the West during that night. In the second part of the night ( $\gamma$ Ari observations) the air masses at altitude were moving along two distinct directions, $\sim 260^{\circ}$ and $\sim 290^{\circ}$, the latter corresponding to the 

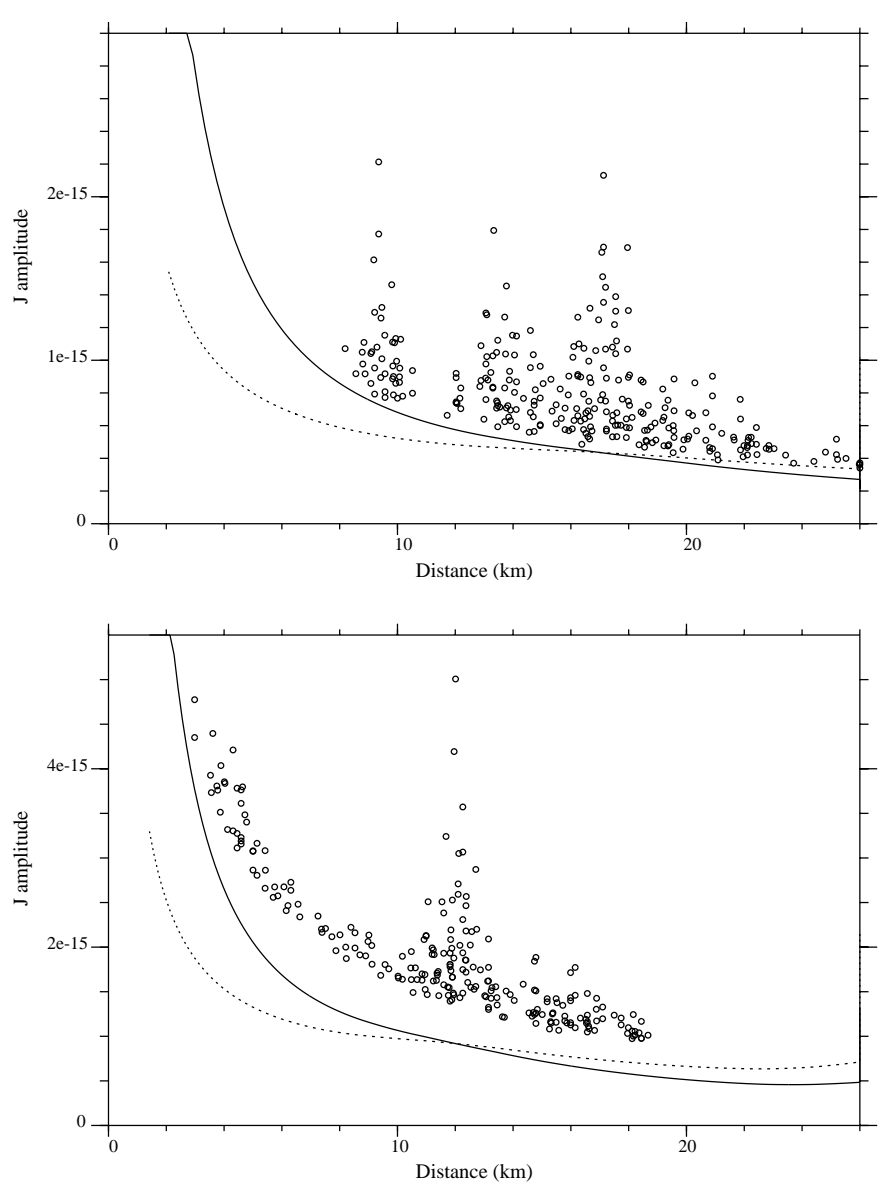

Fig. 12. $\tilde{J}$ amplitude of the components (small circles) detected with the CLEAN algorithm along the different observing sequences. The sensitivity threshold of our SCIDAR observations is shown as a solid curve. The dashed curve is the noise floor as given by (Eq. (14)) for the TBL $(D=2 \mathrm{~m}$ plus central obscuration). a) $\gamma$ Ari (top) with $\sigma_{I}^{2}=0.14, N_{\mathrm{ph}}=110$ photons and $\left.d_{0}=17 \mathrm{~km} ; \mathbf{b}\right) \gamma$ Del (bottom) with $\sigma_{I}^{2}=0.18$, $N_{\mathrm{ph}}=30$ photons and $d_{0}=12 \mathrm{~km}$

direction measured during the first part of the night (with $\gamma$ Del). This rapid change in the atmospheric conditions explains the (transient) co-existence of two wind directions at the same altitude of $\sim 20 \mathrm{~km}$. The arrival of this hot front could also explain the detection of turbulence at very high altitudes $\sim 24 \mathrm{~km}$, which is rather unusual for SCIDAR measurements.

\subsection{Limiting sensitivity of our observations}

As shown in Fig. 12, in the extraction process of turbulence distribution with CLEAN, the components cannot be detected below some sensitivity threshold which is dependent on distance.

Since the size of the components increases with the distance as $d^{1 / 2}$, the curve of detection with constant SNR (solid line in Fig. 12) can be obtained by multiplying the rms noise $\Delta \tilde{J}_{j}$ (as given in Eq. (14)) by $\left(d_{j} / d_{0}\right)^{1 / 2}$ (dashed line in Fig. 12). As in Sect. 2.3, we assume here a constant area for the noise fluctuations which is proportional to the distance $d_{0}$ of the dominant turbulent layer responsible for scintillations.

The model correctly fits variations of sensitivity threshold for $\gamma$ Ari. Actually, a high sensitivity of a few $10^{-16} \mathrm{~m}^{1 / 3}$ has been reached for the upper most turbulent layers. Probing the layers below $\sim 8 \mathrm{~km}$ would have required "generalized" SCIDAR but in that case the scintillation index would have increased because of the contribution of the lower layers. Hence the noise level would have been larger.

Turbulence detection is not as good for $\gamma$ Del and is worse than expected by our model. This can be explained by the low level of the input signal; noise contributions from the detection and recording systems should be added in Eq. (14) for such a weak signal. Another uncertainty concerns the amount of atmospheric absorption which was significant during $\gamma$ Del observations. In Fig. 12, we have assumed an atmospheric transmission of 0.5 for $\gamma$ Del and 0.8 for $\gamma$ Ari, which is only a raw estimate. We also note that this model of sensitivity does not take into account a possible elongation of the noise pattern and the attenuation of the SCIDAR signal due to the finite integration time of each observation (next section), which could explain why the detectivity threshold of $\gamma$ Del observations is not consistent with a constant SNR.

\subsection{Temporal filtering}

The finite integration time $\tau$ of the measurement causes an attenuation of the signal compared to the case of an "instantaneous" measurement which was assumed in Sect. 2. Let $\boldsymbol{V}_{\boldsymbol{j}}$ be the wind velocity of the turbulent layer at a distance $d_{j}$. Assuming a frozen turbulence, the $K$ function defined in (Eqs. (5) and (6)) becomes (Tokovinin 2001):

$$
\begin{aligned}
K\left(\boldsymbol{\rho}, d_{j}, \boldsymbol{V}_{\boldsymbol{j}} \tau\right)= & 1.53 \lambda^{-2} \int f^{-11 / 3} \sin ^{2}\left(\pi \lambda d_{j} f^{2}\right) \\
& \times \operatorname{sinc}^{2}\left(\boldsymbol{f} \cdot \boldsymbol{V}_{\boldsymbol{j}} \tau\right) \exp (2 i \pi \boldsymbol{f} \cdot \boldsymbol{\rho}) \mathrm{d} \boldsymbol{f}
\end{aligned}
$$

This relation is valid as long as $V_{j} \tau$ is much smaller than the diameter of the pupil. Figure 13 shows that the value of the central peak $K\left(\mathbf{0}, d_{j}, \boldsymbol{V}_{\boldsymbol{j}} \tau\right)$ decreases significantly from its theoretical value of one as soon as $V_{j} \tau>0.05 \mathrm{~m}$ with a larger effect for the lower turbulent layers. Using (Eq. (19)), the attenuation was computed for the secondary peaks of $\gamma$ Ari with $\tau=3 \mathrm{msec}$ in Table 3 . The effect is significant for the layers at altitudes in the range 10-15 km which have high velocity winds. Likewise, for $\gamma$ Del, with a turbulent layer at $12 \mathrm{~km}$ and $\tau=2 \mathrm{msec}$, the attenuation factor of the central peak is $\sim 0.5$.

Note that the $K$ attenuation factor of Table 3 is that of the peak, not of the integral of the useful signal in the covariance plane, so that the "Corrected $\tilde{J}$ amplitude" should be taken as an upper bound to the detected turbulence factor.

Using (Eq. (19)), we also computed the deformation of the 2-D profile of the covariance function along two directions: parallel to the wind and perpendicular to it (Fig. 14). It appears that the profile is elongated along 


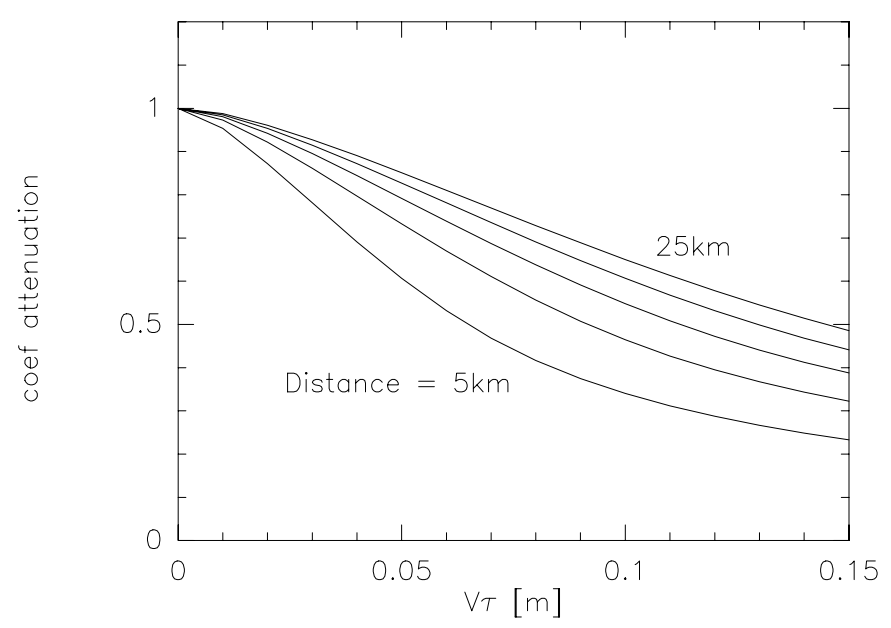

Fig. 13. Attenuation factor of the central peak of the scintillation covariance function due to the finite integration time of the measurements, for distance values of the turbulent layer in the range $5-25 \mathrm{~km}$

Table 3. Parameters of the optical turbulence for the 6 layers detected beyond a distance of $8 \mathrm{~km}$ in Scidar observation of $\gamma$ Ari during the first two minutes of observation. $\tilde{J}$ is in $10^{-15}\left[\mathrm{~m}^{1 / 3}\right]$

\begin{tabular}{lcccccc}
\hline \multicolumn{6}{c}{ Turbulence parameters for the different layers } \\
\hline distance $[\mathrm{km}]$ & 19.6 & 18.8 & 17.1 & 13.7 & 13.1 & 9.4 \\
altitude $[\mathrm{km}]$ & 21.0 & 20.3 & 18.7 & 15.6 & 15.0 & 11.6 \\
wind velocity $[\mathrm{m} / \mathrm{s}]$ & 14.5 & 14.5 & 6.9 & 28.6 & 38.1 & 46.9 \\
wind direction [degree] & 253 & 301 & 247 & 274 & 286 & 276 \\
$\tilde{J}$ amplitude & 2.2 & 3.0 & 11.2 & 3.0 & 5.0 & 10.7 \\
$V_{j} \tau[\mathrm{m}]$ & .043 & .043 & .021 & .086 & .114 & .141 \\
$K$ attenuation & 0.86 & 0.85 & 0.95 & 0.59 & 0.45 & 0.33 \\
Corrected $\tilde{J}$ amplitude & 2.6 & 3.5 & 11.8 & 5.1 & 11.1 & 32.4 \\
\hline
\end{tabular}

the direction of the wind and nearly unaffected perpendicularly. This is visible in the experimental $C_{2}(\boldsymbol{\rho})$ functions: the secondary peaks for altitudes at $\sim 12 \mathrm{~km}$ are elongated along the wind direction (e.g., Fig. 6a). A possible improvement of our 2-D CLEAN algorithm could take into account the actual shape of the profile, elongated along the wind direction, but this is beyond the scope of this paper.

\subsection{Contribution to the seeing from different altitudes}

A seeing of $1.55 \pm 0.05^{\prime \prime}$ was determined just before and after $\gamma$ Ari observations, that is a Fried parameter $r_{0}=0.068 \mathrm{~m}$. Since

$r_{0}=\left[0.423\left(\frac{2 \pi}{\lambda}\right)^{2} \sum_{j} J_{j}\right]^{-3 / 5}$,

the corresponding total optical turbulence factor $\tilde{J}_{\mathrm{T}}=1.4810^{-12} \mathrm{~m}^{1 / 3}$.
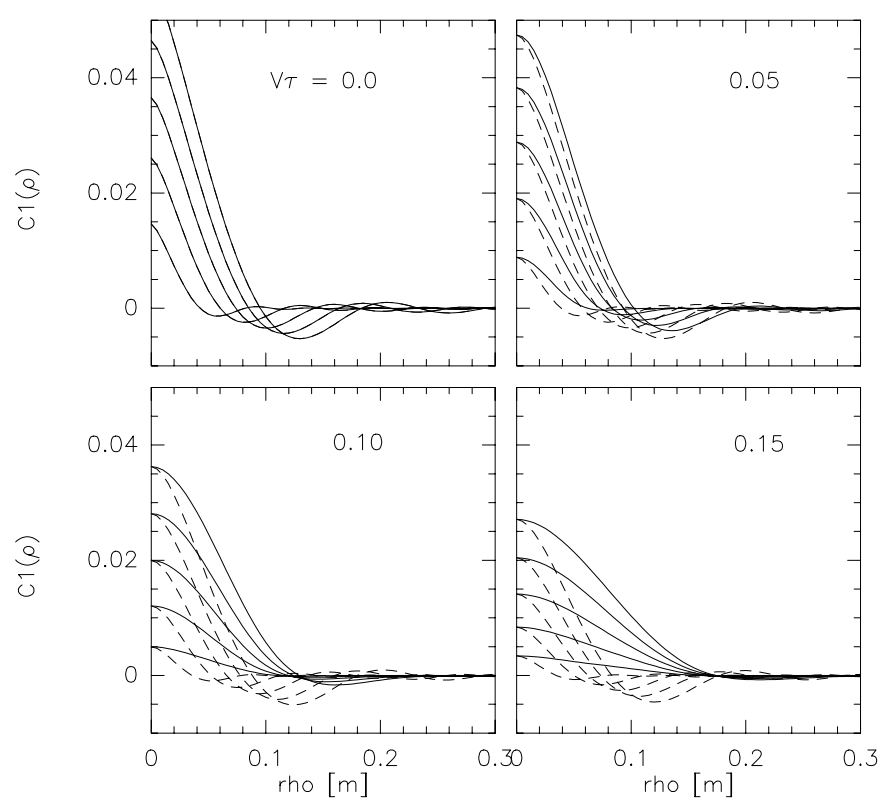

Fig. 14. Profile of the scintillation covariance function for turbulent layers at distances 5, 10, 15, 20 and $25 \mathrm{~km}$. Solid line: profile along the direction of the wind, dashed line: in the perpendicular direction. The value of the product $V_{j} \tau[\mathrm{m}]$ is indicated on each graph

The upper limit of the total content for turbulent layers above $11 \mathrm{~km}$, measured with our observations of $\gamma$ Ari (sum of the last row of Table 3 ) is $\sim 6.610^{-14} \mathrm{~m}^{1 / 3}$, which is less than $5 \%$ of $\tilde{J}_{\mathrm{T}}$. Such a result is consistent with previous findings (Rocca et al. 1974). The contribution of the first kilometers, including the dome seeing and the orographic effects of the site are dominant for the seeing.

\section{Conclusion}

The PISCO speckle camera of Observatoire Midi-Pyrénées has produced SCIDAR measurements for the first time and allowed the derivation of $C_{\mathrm{N}}^{2}$ and wind profiles.

Our implementation of the CLEAN algorithm on the 2-D $C_{2}(\boldsymbol{\rho})$ function showed a higher robustness to the noise which is suitable for the analysis of the time variations. The other traditional MEM approach would also gain from a full use of the 2-D data instead of a slice only. The elongation of the scintillation covariance function due to strong winds in altitude also suggests that a full 2-D processing taking this effect into account would be desirable.

Following the CLEAN approach, an automatic procedure was developed for measuring wind parameters in altitude (velocity and direction) which allowed a full characterization of the turbulence in the upper atmosphere.

The parameters derived from these observations have already been used for simulations of astrometric uncertainties with a dual-field optical interferometer (Daigne et al. 2000). SCIDAR measurements are not sensitive to turbulence scales larger than a few $0.1 \mathrm{~m}$, whereas astrometric uncertainty is mainly dependent on the shape 
of turbulence spectra at much larger scales (a few $10 \mathrm{~m}$ ). A more complete remote investigation of atmospheric turbulence in altitude could be performed with simultaneous observations of differential angle-of-arrival between field stars.

For $\gamma$ Ari, we have observed a rapid change of the turbulence profile with a time-scale of a few minutes only which shows the interest of a continuous turbulence monitoring to improve the performances of future adaptive optics systems in the case of very large telescopes or interferometers.

Acknowledgements. We are indebted to M. Scardia and E. Aristidi for giving us time during their observing run. We are grateful to Météo-France and $\mathrm{Mr}$ Bornuat, director of the Weather Center of Tarbes-Lourdes-Pyrénées Airfield, for kindly providing us with data from the weather unit of Pic du Midi for Table 2. We also thank the technical staff of the "Télescope Bernard Lyot" for providing assistance during the observations. R. Avila was supported by the grant J32412E from CONACyT.

\section{References}

Aristidi, E., Carbillet, M., Prieur, J.-L., et al. 1997, A\&AS, 126,555

Aristidi, E., Prieur, J.-L., Scardia, M., et al. 1999, A\&AS, 134, 545

Avila, R. 1998, Ph.D. Thesis, Nice University, France

Avila, R., Vernin, J., \& Masciadri, E. 1997, Appl. Opt., 36, 7898

Avila, R., Vernin, J., \& Cuevas, S. 1998, PASP, 110, 1106

Avila, R., Vernin, J., \& Sánchez, L. J. 2001, A\&A, 369, 364

Caccia, J. L., Azouit, M., \& Vernin, J. 1987, Appl. Opt., 26, 1288
Colavita, M. M. 1994, A\&A, 283, 1027

Colavita, M. M., Wallace, J. K., Hines, B. E., et al. 1999, ApJ, 501,505

Coulman, C. E., Vernin, J., \& Fuchs, A. 1995, Appl. Opt., 34, 5461

Daigne, G., Prieur, J.-L., \& Avila, R. 2000, Astronomical site evaluation in the visible and radio range, 13-17 November 2000, Marrakech, Morocco, ASP Conf. Ser., submitted

Dubosclard, G., Hecquet, J., Coupinot, G., \& Sauvageot, H. 1984, A\&A, 140, 273

Fried, D. L. 1966, JOSA, 56, 1372

Fuchs, A., Tallon, M., \& Vernin, J. 1998, PASP, 110, 86

Hecquet, J., \& Klauss, V. 1989, A\&A, 225, 585

Klückers, V. A., Wooder, N. J., Nicholls, T. W., et al. 1998, A\&AS, 130, 141

Lane, B. F., Colavita, M. M., Boden, A. F., \& Lawson, P. R. 2000, SPIE, 4006, 452

Prieur, J.-L., Koechlin, L., André, C., Gallou, G., \& Lucuix, C. 1998, Exper. Astron., vol. 8, Issue 4, 297

Prieur, J.-L., Oblak, E., Lampens, P., et al. 2000, A\&A, 367, 865

Rocca, A., Roddier, F., \& Vernin, J. 1974, JOSA, 64, 1000

Roddier, F. 1981, The effects of atmospheric turbulence in optical astronomy in Progress in Optics, ed. E. Wolf, XIX, 281

Scardia, M., Prieur, J.-L., Aristidi, E., \& Koechlin, L. 2000, ApJS, 131, 561

Shao, M. \& Colavita, M. M. 1992, A\&A, 262, 353

Schwarz, U. J. 1978, A\&A, 65, 345

Tatarskii, V. I. 1961, Wave propagation in a Turbulent Medium (Dover ed., New-York)

Tokovinin, A. A. 1997, VLT-TRE-UNI-17416-0003 (ESO, Garching)

Tokovinin, A. A. 2001, Appl. Opt., in press

Vernin, J. 1984, Report, Nice University, France

Vernin, J., \& Azouit, M. 1983, J. Opt. (Paris), 14, 131 